\title{
First Observations with Multi-Pupil Integral Field Spectrograph on 4-meter Mayall Telescope
}

\author{
V. L. Afanasiev ${ }^{1}$, and V. V. Vlasiouk ${ }^{1}$ \\ Special Astrophysical Observatory of Russian Academy of Sciences, \\ Nyzhnij Arkhyz, Russia,357147
}

R. F. Green

National Optical Astronomy Observatories, Tucson, AZ 85719, USA

\section{Introduction}

First attempts to get spectra from extended areas were in the middle of 80-th, using fibers techniques : DensePack (Barden,Scott,1986), SILFID (Vanderriest and Lemonnier,1988). Bundle of fibers were put at the focus of the telescope and the other ends of fibers rearranged to produce a long slit of the spectrograph.

Another method of bidimensional spectroscopy, not using optical fibers, was proposed by Prof. G.Courtes (Courtes et al,1988). He recommended to put behind the telescope focal plane a bidimensional array of microlenses and to project on it an enlarged image. The lens array separates continuous input image and forms an array of micro-pupils that are images of the main mirror illuminating each lens. They create an input image for a classical spectrograph instead of a normal slit. Using of square lenses allows to achieve correct data sampling, and such scheme is ideal for spectrophotometry, allowing integration of total energy from the observed sky area.

The main advantages of this method are continuous sampling of the analyzed sky area and a better transmission. One of it's disadvantages is incomplete filling of detector by spectra, especially in low-dispersion mode.

\section{Main characteristics of the instrument}

Following this idea, a spectrograph had been built in the SAO of Rus. Ac. Sci. in 1990 and now is using during spectral observations of galaxies' nuclei on the 6-meter telescope (Afanasiev, Sil'chenko,1991, Sil'chenko et al.,1992, and several contributions at this Colloquium)

One of the conditions for the development of analog scheme for Mayall's 4meter telescope was to fit enlargers and micro-lens array into RC spectrograph instead of standard slit. We designed a scheme featuring auxiliary mirrors, that allowed an acceptable total length of the implemented block.

\footnotetext{
${ }^{1}$ Viriting Astronomer, Kitt-Peak National Observatory. KPNO is operated by AURA, Inc. under cooperative agreement with the National Science Foundation
} 
SAO specially produced a microlens array for 4-meter telescope. It consists of 10 by $162.6 \mathrm{~mm}$ square lenses. Focal ratio of each lens is $\mathrm{f} / 7$, typical accuracy of individual lens positions is better than $30 \mu \mathrm{m}$.

The set of remotely changed enlargers achieves spatial sampling of data from 1 " $\left(8^{x}\right.$ enlarger $)$ to 0.25 " $\left(30^{x}\right.$ enlarger $)$ per lens. The size of covered field also is proportionally changed : from $10^{\prime \prime} \times 16^{\prime \prime}$ to $2.5^{\prime \prime} \times 4^{\prime \prime}$.

The spectral characteristics of MPIFS are defined by the parameters of the RC spectrograph with one advantage: the size of individual micro-pupil, defining spectral resolution, is smaller than the standard slit width on RC ( 300 $\mu \mathrm{m})$. This size depends on used enlargement and is between 100 and $200 \mu \mathrm{m}$.

\section{Observations}

During 2 nights nuclei of 3 Seyfert galaxies were observed with MPIFS: NGC 3516, NGC 5548 and NGC 6814. We used for data acquisition CCD T2KB $(2048 \times 2048 \mathrm{pix})$ in combination with Simmonson camera. We choose this camera in attempt to reach a better spectra separation.

The angular sampling of our data was 0.75 "/lens, spectral samping was $0.37 \AA / \mathrm{pix}$ with grating KPC-24 in second order and $0.95 \AA /$ pix with grating KPC-007 in first order. Detected wavelength range was $4500-5200 \AA$ and 3600 $5500 \AA$, accordingly.

Typical integration time for each objects was 1.5-2 hours, which was divided on sub-exposures $30 \mathrm{~min}$ long to suppress cosmic ray events on CCD accumulations. Typical image FWHM during our observations was 1.2 - $1.5 "$.

Total efficiency of MPIFS, estimated from data obtained on the standard source $P G 1708+602$, equals to $3.5 \%$ at $5100 \AA$. This value is consistent with efficiency of HYDRA spectrograph, where the same combination CCD+camera is using.

\section{Data Reduction and Analysis}

Spectral images were processed as usually, with IRAF: bias subtraction, flatfielding and cosmic rays suppression have been done. Next steps of data processing have been executed with specialized software, developed in SAO and Marseille observatories. It included: spectra extraction after overlapped data separation, wavelength calibration, measurements of spectral features parameters.

Preliminary analysis of [OIII] $\lambda 5007 \AA$ emission line profiles in central part of NGC 3516, obtained with high spectral resolution $(0.37 \AA /$ pix $)$, showed existence of two components, previously described by Mulchaey et al., 1992. On our opinion, it demonstrates presence in center of this galaxy of a helical structure, located on the sides of a cone whose axis coincides with the radio jet direction.

In interpretation of observed data it may be very important to know the characteristics of the galaxy stellar disk rotation. We used spectral data in the wavelength range $3900-4800 \AA$ with $1 \AA$ sampling, including the strongest stellar absorption lines, in order to avoid confusion from emission lines to get velocity field for stellar population. Using of cross-correlation techniques ( Tonry, 
Davis,1979) allowed us to build this field with a mean accuracy of about 15-20 $\mathrm{km} / \mathrm{sec}$.

\section{References}

Afanasiev, V.L., Sil'chenko, O.K. : 1991, Astrofiz.Issled (Izv.SAO), 34(in Russian).

Barden, S.G., Scott, K. : 1986, BAAS, 18, 951.

Courtes, G., Georgelin, Y., Bacon, R., Monnet, G., Boulesteix, J. 1988, in Instrumentation for Ground-Based Optical Astronomy : Present and Future, ed. L. Robinson, 266.

Mulchaey, J.S., Tsvetanov, Z., Wilson, A.S., Perez-Fournon, I.: 1992, ApJ,394, 91

Sil'chenko, O.K., Afanasiev, V.L., Vlasiuk, V.V. 1992, AZh, 69,1121 (in Russian).

Tonry, J., Davis, M. 1979, AJ, 84, 1511

Vanderriest, C., Lemonnier, J.-P. : 1988, in Instrumentation for Ground-Based Optical Astronomy : Present and Future, ed. L.Robinson, 305 